\title{
Pathophysiological analysis and strategy for stercoral perforation of the colon
}

\author{
Koichi Sato $^{{ }^{*}}$, Hiroshi Maekawa ${ }^{1}$, Mutsumi Sakurada ${ }^{1}$, Hajime Orita ${ }^{1}$, Tomoaki Ito ${ }^{1}$, \\ Yoshihiro Komatsu ${ }^{1}$, Fumiko Hirata ${ }^{1}$, Ryo Wada ${ }^{2}$ \\ ${ }^{1}$ Department of Surgery, Juntendo Shizuoka Hospital, Juntendo University School of Medicine, Shizuoka, Japan \\ ${ }^{2}$ Department of Pathology, Juntendo Shizuoka Hospital, Juntendo University School of Medicine, Shizuoka, Japan \\ Email: ${ }^{*}$ kou-sato@chive.ocn.ne.jp
}

Received 6 January 2012; revised 30 January 2012; accepted 8 February 2012

\begin{abstract}
Perpose: In order to establish the pathophysiological features and strategy for stercoral perforation of the colon, we herein analyze a series of stercoral perforation of the colon. Method: Ten patients were diagnosed with stercoral perforation. Clinical features, primary diseases, triggers, causative bacteria in ascites, postoperative complications, pathological features, severity of the disease, and effect of direct hemoperfusion with polymyxin B immobilized fiber (PMX-DHP) were investigated. Results: Nine patients had a long history of serious and chronic constipation and 7 patients had hypertension. Causative bacteria in ascites during the operation were most commonly Escherichia coli. There were a lot of severe postoperative complications such as sepsis, disseminated intravascular coagulation, and acute lung injury. With regard to the microscopic findings of the perforation site, the intestinal wall showed severe nonspecific inflammatory changes, including an increase of mononuclear cells in the lamina propria. There were 4 hospital deaths, so the mortality rate was $40 \%$. APACHEII and SOFA score were high postoperation and 24 hours after the operation. PMX-DHP was performed in 8 cases of severe conditions of stercoral perforation of the colon. Because the catecholamine index improved within 24 hours, four of 8 cases were rescued. Conclusion: Most of the patients with stercoral perforation of the colon had severe postoperative complications. The severity of the disease was extremely high, therefore, early diagnosis based on pathophysiological features and comprehensive therapies including PMX-DHP were necessary for strategy of treating stercoral perforation of the colon.
\end{abstract}

Keywords: Stercoral Perforation of the Colon; APACHE-II Score; SOFA Score; Polymyxin B

*Corresponding author.
Immobilized Fiber (PMX-DHP)

\section{INTRODUCTION}

Stercoral perforation of the colon is rare with less than 80 cases reported in the world literature to date [1-4]. The diagnosis of stercoral perforation is poor with postoperative mortality rates of about 35\% - 40\% [5,6]. Early diagnosis and treatment should improve survival [7]. In order to establish the pathophysiological features and strategy for stercoral perforation of the colon, we herein analyze a series of 10 cases of stercoral perforation of the colon experienced in our hospital, and investigate the effectiveness of direct hemoperfusion with polymyxin (PMX) B immobilized fiber (PMX-DHP) by which we have obtained the improvement of survival rates for the infectious diseases of the abdominal cavity.

\section{PATIENTS AND METHODS}

\subsection{Patients}

Between October 1988 and May 2011, all 217 cases of perforation of the large intestine experienced in our hospital were prospectively recorded on a detailed database. Of all cases of perforation of the large intestine, 10 patients of stercoral perforation, diagnosed in accordance with the diagnostic criteria proposed by Huttunen et al. [8], were investigated.

The etiology of perforation was ruptured diverticulitis in 78 patients, cancer in 58 patients, iatrogenic colon perforation related to colonoscopy procedure in 28 patients, idiopathic colon perforation in 15 patients, 13 patients with traumatic colon perforation, other colon perforation in 15 patients, and 10 patients were diagnosed with stercoral perforation in accordance with the diagnostic criteria proposed by Huttunen et al. [8].

\subsection{Methods}

The following items, that is, clinical features (average age, gender, presence of pneumoperitoneum, presence of 
shock, times between perforation and operation), primary diseases and triggers of stercoral perforation, intra-operative findings and operative procedures, causative bacteria in ascites during the operation, pathological features, postoperative complications, severity of the disease (expressed with APACHE-II and SOFA scores), severity of the disease according to outcome, and the effect of direct hemoperfusion with polymyxin (PMX) B immobilized fiber (PMX-DHP) were investigated in the 10 patients with stercoral perforation.

APACHE-II score was calculated by the method of Knaus et al. [9], and SOFA score was also calculated by the method of Vincent et al. [10].

PMX-DHP was performed immediately after the operation using a double-lumen catheter within the femoral vein for blood access. The second PMX-DHP was performed the next day in the severe cases that still demonstrated SIRS after the first treatment. Using PMX-20R (TREMIXIN, Toray; Tokyo, Japan) as the hemopurification column, extracorporeal circulation was conducted for 2 hours at a blood flow rate of $80-120 \mathrm{ml} / \mathrm{min}$. Nafamostat mesilate (30 - $40 \mathrm{mg} / \mathrm{hr}$ ) was continuously administered as the anticoagulant.

\subsection{Statistical Analysis}

Data are presented as the mean \pm standard deviation (SD). Differences were assayed for statistical significance by Mann-Whitney's U-test. A P value of $<0.05$ was considered as statistically significant.

\section{RESULTS}

All the 10 patients were women aged between 62 - 94 years. Their averaged age was very high, 79.1 years old. Four patients were found to have pneumoperitoneum on a standing chest roentgenogram and/or abdominal CT scan, and two patients experienced states of shock. Times between perforation and operation were 6 - 96 hours. The average time was 23 hours.

The primary diseases were shown in Table 1. Nine patients had a long history of serious and chronic constipation and 7 patients had hypertension (Table 1). The triggers of disease were cathartics (4 patients) and enema (3 patients).

All patients underwent emergency laparotomy with a preoperative diagnosis of hollow organ perforation. Seven patients underwent Hartmann's operative procedure with colostomy and another three patients were treated with exteriorization.

In 10 patients where the site of perforation was documented, the most common site was the sigmoid colon (70 percent). The next commonest was the descending colon (30 percent).

The causative bacteria in ascites during the operation were shown in Table 2. All of these causative bacteria belong to the gram-negative bacilli (Table 2).

The gross and microscopic findings of seven resected specimens were shown in Table 3. Perforations were appeared as a single lesion and one was a double lesion that seemed to have been punched out. The intestinal wall showed transmural necrosis. In three cases, fecal particles broke into the necrotic wall of the intestine (Table 3). The typical ovoid perforation of the colon was shown in a figure (Figure 1).

The postoperative complications were shown in Table 4. There were a lot of severe complications (Table 4).

There were 4 hospital deaths, so the mortality rate was $40 \%$. The conditions of stercoral perforation of the colon were severe. APACHE-II score of stercoral perforation of the colon was very high preoperation, postoperation, and 24 hours after the operation. SOFA score was also high postoperation and 24 hours after the operation (Figure 2). Moreover, APACHE-II score was higher in the fatality group than the survival group. SOFA score was, however, the same in the two groups (Figure 3 ).

Direct hemoperfusion with polymyxin (PMX) B immobilized fiber (PMX-DHP) was performed in 8 cases of severe conditions of stercoral perforation of the colon.

Table 1. Primary diseases.

\begin{tabular}{ll}
\hline \multicolumn{1}{c}{ Primary disease } \\
\hline Constipation & 9 cases \\
Hypertension & 7 cases \\
Myocardial infarction, Angina Pectoris & 2 cases \\
Arrythmia & 1 cases \\
Cerebral infarction & 2 cases \\
Cerebral aneurysm & 2 cases \\
Parkinsonism & 1 cases \\
Chronic renal failure & 2 cases \\
Rheumatoid arthritis (Steroid) & 1 cases \\
Diabetes mellitus & 1 cases \\
\hline
\end{tabular}

Table 2. Causative bacteria in ascites during operation.

\begin{tabular}{ll}
\hline \multicolumn{1}{c}{ Causative bacterium } & \\
\hline Escherichia coli & 5 cases \\
Klebsiella pneumoniae & 3 cases \\
Enterococcus faecalis & 3 cases \\
Bacteroides & 2 cases \\
Pseudomonas aeruginosa & 2 cases \\
Enterobacter cloacae & 1 cases \\
Enterococcus faecium & 1 cases \\
Peptostreptococcus & 1 cases \\
Other & 1 cases \\
\hline
\end{tabular}


Table 3. Pathological features.

\begin{tabular}{|c|c|c|c|c|c|c|c|}
\hline & 1 & 2 & 3 & 4 & 5 & 6 & 7 \\
\hline Age & 85 & 62 & 81 & 94 & 86 & 82 & 74 \\
\hline Gender & female & female & female & female & female & female & female \\
\hline Site of perforation & S & $\mathrm{S}$ & $\mathrm{S}$ & $\mathrm{D}$ & $\mathrm{S}$ & S & $\mathrm{D}$ \\
\hline Number of perforation & single & single & multiple & single & single & single & single \\
\hline Diameter of perforation & $8 \mathrm{~cm}$ & $3 \mathrm{~cm}$ & $3 \mathrm{~cm}$ & $3 \mathrm{~cm}$ & $3.5 \mathrm{~cm}$ & $3.5 \mathrm{~cm}$ & $3 \mathrm{~cm}$ \\
\hline Shape of perforation & round & round & ovoid & ovoid & ovoid & ovoid & ovoid \\
\hline Inflammation at perforation site & severe & severe & severe & severe & severe & severe & severe \\
\hline Necrosis at perforation site & transluminal & transluminal & transluminal & transluminal & transluminal & transluminal & transluminal \\
\hline Feces into bowel wall & none & present & none & none & present & none & present \\
\hline Atrophy of bowel wall & present & none & none & none & none & none & none \\
\hline Granulation of bowel wall & present & present & none & none & none & none & none \\
\hline
\end{tabular}

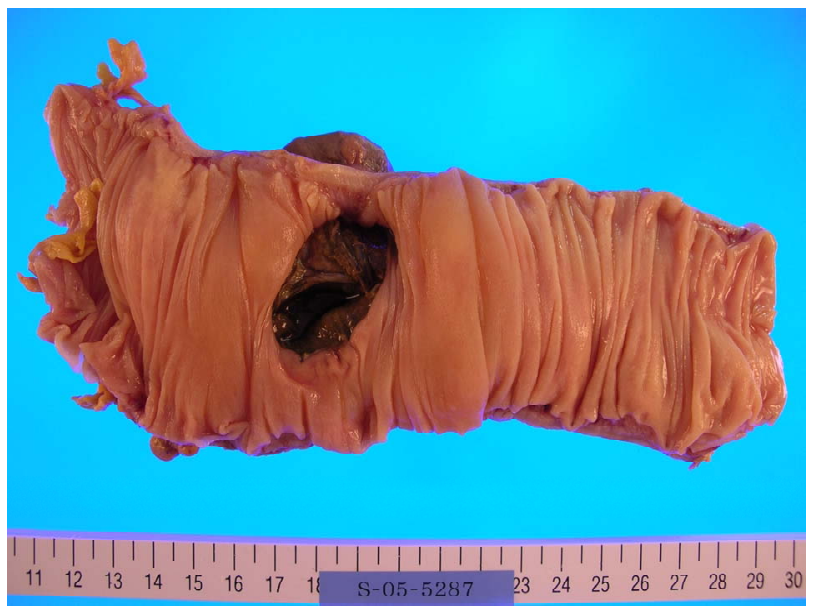

Figure 1. Ovoid perforation of the colon.

Table 4. Postoperative complications.

\begin{tabular}{ll}
\hline \multicolumn{1}{c}{ Postoperative complication } \\
\hline Sepsis & 3 cases \\
Disseminated intravascular coagulation & 2 cases \\
Acute lung injury & 1 cases \\
Acute renal failure & 1 cases \\
Liver dysfunction & 1 cases \\
Atrial fibrillation & 1 cases \\
Ileus & 1 cases \\
Wound infection & 2 cases \\
\hline
\end{tabular}

Because the catecholamine index improved within 24 hours, four of 8 cases were rescued (Table 5).

\section{DISCUSSION}

Stercoral perforation of the colon is considered to be an extremely rare disease. Stercoral perforation of the colon was first reported by Berry [11] to the Pathological
Table 5. PMX-DHP and CAI.

(a)

\begin{tabular}{cc}
\hline Mortality & $4 / 10(40 \%)$ \\
PMX & $8 / 10(80 \%)$ \\
Mortality in PMX & $4 / 8(50 \%)$ \\
\hline
\end{tabular}

(b)

\begin{tabular}{cccc}
\hline & Pre-PMX & Post-PMX & 24 hours after PMX \\
\hline CAL & $26.1 \pm 45.4$ & $27.6 \pm 46.7$ & $8.2 \pm 8.3$ \\
\hline
\end{tabular}

CAI (catecholamine index $)=($ dopamine dose $\times 1)+($ dobutamine dose $\times 1)$ $+($ adrenaline dose $\times 100)+($ noradrenaline dose $\times 100) ; \mathrm{P}<0.01$ vs. preand post-PMX-DHP.

Society of London in 1894. According to a recent study, which was one of the largest studies ever carried out by a single institution, the incidence of stercoral perforation of the colon comprised $1.2 \%$ of all cases requiring emergency colorectal surgery and $3.2 \%$ of all those involving colonic perforations [8].

This disease is defined as "perforation of the bowel due to pressure necrosis from fecal masses" [5]. The resulting criteria for the diagnosis of stercoral perforation of the colon, as opposed to the clinical criteria of Huttunen et al. [12], are as follows: 1) the colonic perforation is round or ovoid, exceeding $1 \mathrm{~cm}$ in diameter, and is orientated antimesenterically; 2) fecalomas are present within the colon, protruding through the perforation site, or lying within the abdominal cavity; and 3) pressure necrosis or ulcer and chronic inflammatory reaction are microscopically present around the perforation site.

Severe chronic constipation is considered to be the main causative factor in the development of stercoral perforation of the colon [1,5,7,12-15]. The fecalomas exert a constant pressure on the bowel wall and lead to pressure necrosis of the mucosa. Several factors help explain why the left hemicolon is predisposed to stercoral perforation of the colon. The sigmoid and rectosigmoid 


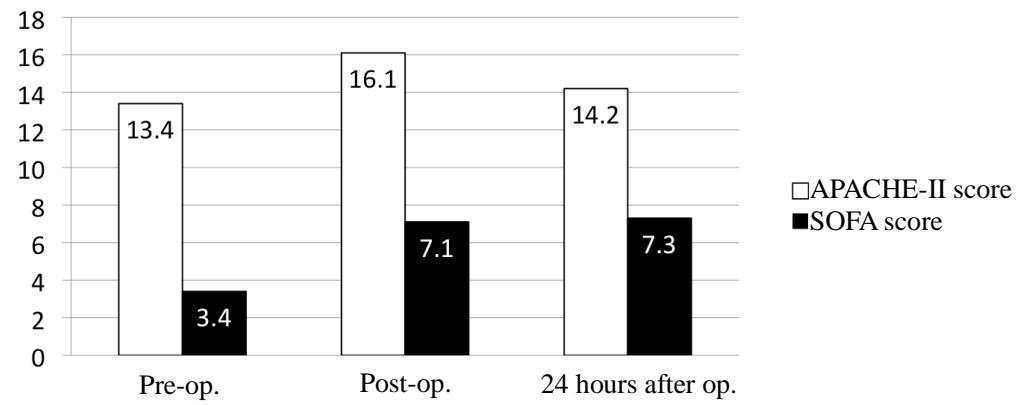

Figure 2. Severity of the disease: APACHE-II score of stercoral perforation of the colon was very high preoperation, postoperation, and 24 hours after the operation. SOFA score was also high postoperation and 24 hours after the operation.

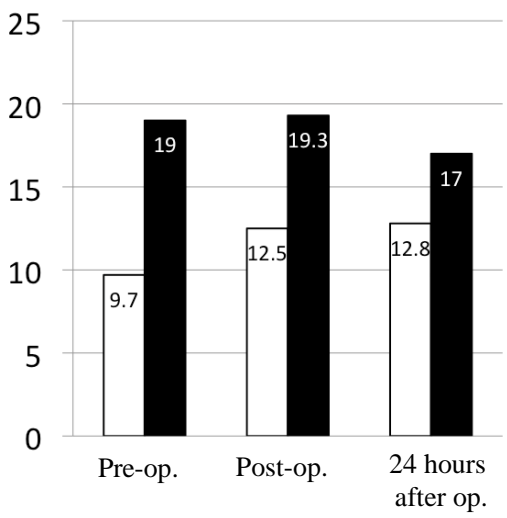

(a)

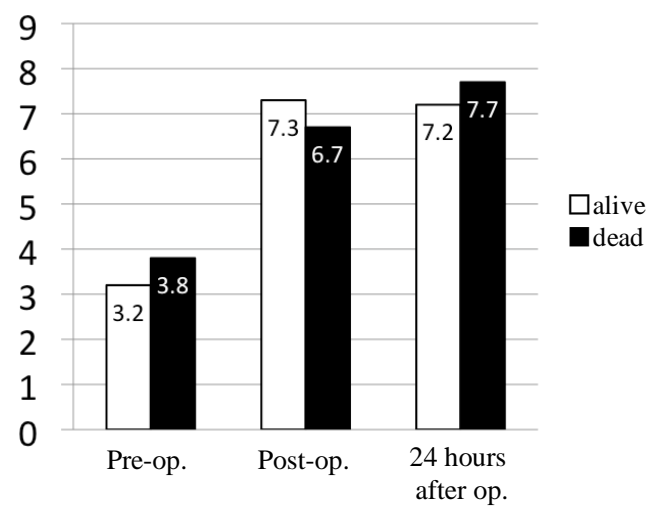

(b)

Figure 3. Severity of the disease according to outcome: (a) APACHE-II score was higher in the fatality group than the survival group; (b) SOFA score was, however, the same in the two groups.

are the narrowest segments of the colon. The transport of fecalomas into the rectum needs a further increase of the intratuminal pressure by contraction of the colonic smooth muscles. This may compromise the microcirculation of the bowel wall and result in stercoral perforation.

Serpell and Nicholls [5] reported that, of a total of 64 cases of stercoral perforation reviewed, only 39 (61 percent) presented with a long history of chronic constipation preceding the stercoral perforation and five patients exhibited chronic renal failure. Of 43 patients subjected to plain radiography of the chest and abdomen, 23 patients (53 percent) had pneumoperitoneum, and correct diagnosis was only made before surgery in seven patients. They also reported that, in 57 patients where the site of perforation was documented, the most common site was the sigmoid colon (47 percent). The next commonest was the rectosigmoid (30 percent) followed by the cecum (9 percent), transverse colon ( 7 percent), descending colon (5 percent), and splenic flexure (2 percent). There was a single perforation in 79 percent of cases and multiple perforations in the remainder. Similarly in our cases, nine patients (90 percent) had a long history of serious and chronic constipation and the most common site of per- foration was the sigmoid colon (70 percent).

Grinvalsky and Bowenman [16] characterized the pathology of stercoral ulcers. The lesion is an ischemic pressure necrosis due to hard scybala producing irregular, geographically outlined ulcers, which conform to the contour of the impacted scybala. The ulcers are depressed below the mucosal level, often multiple and histologically show ischemic pressure necrosis and an inflammatory reaction. A stecoral ulcer precedes a stecoral perforation. A rounded or ovoid perforation may occur in the center of the ulcer where thinning is maximal [17, 18].

Mortality because of stercoral perforation of the colon is reported to be high $[5,7,19,20]$. Similarly in our cases, four patients (40 percent) had been dead. Maurer et al., however, provided several factors for the favorable outcome as follows: the patients were 50 years old or younger, a laparotomy was performed within a few hours after admission under the diagnosis of perforation within the gastrointestinal tract, the affected colon segment was always generously resected, intraoperatively extensive peritoneal lavage with Ringer's solution followed, Hartmann's operation was the preferred procedure, and no 
primary anastomosis was performed. In our cases, seven patients were also received Hartmann's operation. However the motality rate was very high because almost all patients were very old.

The usefulness of PMX-DHP for abdominal sepsis was demonstrated by two prospective randomized controlled trials in Europe [21,22] and one meta-analysis [23]. A systematic review [22] found positive effects of PMX-DHP on mean arterial pressure (MAP) and dopamine/dobutamine use, $\mathrm{PaO}_{2} / \mathrm{FiO}_{2}$ ratio, endotoxin removal, and mortality. Pooled mortality rates were $61.5 \%$ in the conventional therapy group and $33.5 \%$ in the PMX group. In the pooled estimate, PMX-DHP appeared to significantly reduce mortality compared with conventional medical therapy. In a prospective randomized controlled trial (RCT) of surgical patients with septic shock and severe sepsis induced by abdominal sepsis (EUPHAS study) [23], PMX-DHP was effective in improving 28day and hospital survival, blood pressure, vasopressor requirement, and degree of organ failure as indicated by SOFA score when added to conventional medical treatment. The 28-day crude mortality was 32\% in the PMX group and $53 \%$ in the conventional therapy group. The reason for the mortality rate of stercoral perforation of the colon being extremely high is that severity expressed by APACHE-II score and SOFA score is high in this disease. PMX-DHP was effective in improving survival for the reasons mentioned above. In our cases, four of 8 cases were rescued though APACHE-II and SOFA score were very high because the catecholamine index improved within 24 hours

\section{CONCLUSION}

Most of the patients with stercoral perforation of the colon had severe postoperative complications. The severity of the disease was extremely high, therefore, early diagnosis based on pathophysiological features and comprehensive therapies including PMX-DHP were necessary for strategy of treating stercoral perforation of the colon.

\section{REFERENCES}

[1] Bauer, J.J., Weiss, M. and Dreiling, D.A. (1972) Stercoraceous perforation of the colon. Surgical Clinics of North America, 52, 1047-1053.

[2] Dubinsky, I. (1996) Stercoral perforation of the colon: Case report and review of the literature. Jourmal of Emergency Medicine, 14, 323-325. doi:10.1016/0736-4679(96)00044-3

[3] Gul, Y.A., Waldron, D.J. and O’Connell, P.R. (1997) Stercoral perforation associated with parathyroid adenoma. Irish Medical Journal, 90, 20.

[4] DeJong, J.L., Cohle, S.D. and Busse, F. (1996) Fetal stercoral ulcer perforation: Case report. American Journal of
Forensic Medecine and Pathology, 17, 58-60. doi:10.1097/00000433-199603000-00010

[5] Serpell, J.W. and Nicholls, R.J. (1990) Stecoral perforation of the colon. British Journal of Surgery, 77, 13251329. doi:10.1002/bjs. 1800771204

[6] Strutynsky, N. and Orron, D. (1987) Stercoral perforation of the descending colon appearing radiographically as pneumomediastinum. Mount Sinai Journal of Medicine, 54, 436-439.

[7] Gekas, P. and Schuster, M. (1981) Stercoral perforation of the colon: Case report and review of the literature. Gastroenterology, 80, 105.

[8] Maurer, C.A., Renzulli, P., Mazzucchelli, L., et al. (2000) Use of accurate diagnostic criteria may increase incidence of stercoral perforation of the colon. Diseases of Colon and Rectum, 43, 991-998. doi:10.1007/BF02237366

[9] Knaus, W.A., Draper, E.A., Wagner, D.P., et al. (1985) APACHE-II score: A severity of disease classification system. Critical Care Medicine, 13, 818-829. doi:10.1097/00003246-198510000-00009

[10] Vincent, J.L., Moreno, R., Takara, J., et al. (1996) The SOFA (Sepsis-related Organ Failure Assessment) score to describe organ dysfunction/failure. Intensive Care Medicine, 22, 707-710. doi:10.1007/BF01709751

[11] Berry, J. (1894) Dilatation and rupture of sigmoid flexure [short report]. British Medical Journal, 1, 301.

[12] Carter, D.C. and Kirkpatrick, J.R. (1973) Stercoral perforation of the sigmoid colon. British Journal of Surgery, 60, 61-63. doi:10.1002/bjs.1800600117

[13] Sharma, M. and Agrawal, A. (2010) Case report: Stercoral sigmoid colonic perforation with fecal peritonitis. Indian Journal of Radiology and Imaging, 20, 126-128. doi:10.4103/0971-3026.63051

[14] Wald, A. (2008) Management and prevention of fecal impaction. Current Gastroenterology Reports, 10, 499501. doi:10.1007/s11894-008-0091-y

[15] Nam, J.K., Kim, B.S., Kim, K.S., et al. (2010) Clinical analysis of stercoral perforation of the colon. Korean Journal of Gastroenterology, 55, 46-51. doi:10.4166/kjg.2010.55.1.46

[16] Grinvalsky, H.T. and Bowenman, C.I. (1959) Stercoraceous ulcers of the colon: Relatively neglected medical and surgical problem. The Journal of the American Medical Association, 171, 1941-1946. doi:10.1001/jama.1959.03010320031008

[17] Lyon, D.C. and Sheiner, H.J. (1969) Idiopathic rectosigmoid perforation. Surgery Gynecology and Obstetrics, 128, 991-1000

[18] Barardi, R.S., Lee, S.S., Chen, H.P., et al. (1987) Stercoraceous and spontaneous perforation of the colon. International Surgery, 72, 235-240.

[19] Guyton, D.P., Evans, D. and Schreiber, H. (1985) Stercoral perforation of the colon. Concepts of operative management. American Journal of Surgery, 51, 520-522.

[20] Sung, J.F., Salvay, H.B., Hansman, M.F., et al. (2009) Stercoral perforation of the colon with favorable pregnancy outcome. Obstetrics and Gynecology, 113, 491- 
492.

[21] Vincent, J.L., Laterre, P.F., Cohen, J., et al. (2005) A pilot-controlled study of a polymyxin B-immobilized hemoperfusion cartridge in patients with severe sepsis secondary to intra-abdominal infection. Shock, 23, 400405. doi:10.1097/01.shk.0000159930.87737.8a

[22] Cruz, D.N., Perazella, M.A., Bellomo, R., et al. (2007)
Effectiveness of polymyxin B-immobilized fiber column in sepsis; a systematic review. Critical Care, 11, 47. doi:10.1186/cc5780

[23] Cruz, D.N., Antonelli, M., Fumagalli, R., et al. (2009) Early use of polymyxin B hemoperfusion in abdominal septic shock. The Journal of the American Medical Association, 301, 2445-2452. doi:10.1001/jama.2009.856 